\title{
Characterization of the Toxoplasma gondii hsp60 gene sequences from different hosts and geographical locations
}

J. Lu' ${ }^{1,2}$, D.H. Zhou ${ }^{1}$, J. Chen ${ }^{1}$, N.Z. Zhang ${ }^{1}$, R.A. Wang ${ }^{1,2}$, Y.B. Weng ${ }^{2}$ and X.Q. $\mathbf{Z h u}^{1}$

${ }^{1}$ State Key Laboratory of Veterinary Etiological Biology,

Key Laboratory of Veterinary Parasitology of Gansu Province, Lanzhou Veterinary Research Institute,

Chinese Academy of Agricultural Sciences, Lanzhou, Gansu Province, China ${ }^{2}$ Department of Parasitology, College of Veterinary Medicine,

South China Agricultural University, Guangzhou, Guangdong Province, China

Corresponding author: X.Q. Zhu

E-mail: xingquanzhu1@hotmail.com

Genet. Mol. Res. 13 (3): 6906-6911 (2014)

Received July 10, 2013

Accepted February 10, 2014

Published August 29, 2014

DOI http://dx.doi.org/10.4238/2014.August.29.13

ABSTRACT. The intracellular protozoan Toxoplasma gondii is one
of the most successful parasites, with the ability to invade all warm-
blooded animals, including humans. T. gondii heat shock protein 60
(TgHSP60) plays an important role in intracellular survival and in the
differentiation of the parasite, and is also recognized as being associated
with its virulence. In the present study, we examined sequence variation
in the $h s p 60$ coding region among five $T$. gondii isolates from different
hosts and geographical regions, which were compared with the
corresponding sequences of strains ME49, $76 \mathrm{~K}$, and GT1 available
in the ToxoDB databases. The length of the T. gondii hsp60 sequence
was 1728 bp for all strains, and the A+T content ranged from 41.96 to
$42.13 \%$. The sequence alignment of the 8 . gondii strains identified
20 variable positions $(0-1.44 \%)$ and showed $1.16 \%$ overall sequence 
variation, suggesting a relatively considerable sequence diversity. Phylogenetic analysis of hsp60 sequences using Bayesian inference and maximum parsimony differentiated the two major clonal lineage types into their respective clusters, and thus separated atypical strains from classical genotypes. The results of the present study suggested that the coding region of the $h s p 60$ gene may represent a novel genetic marker for intraspecies phylogenetic analyses of $T$. gondii.

Key words: Toxoplasma gondii; Toxoplasmosis; Sequence variation; Heat shock protein 60; Phylogenetic analysis

\section{INTRODUCTION}

As an obligate intracellular protozoan pathogen, Toxoplasma gondii has worldwide distribution and can invade virtually all warm-blooded animals and humans, leading to toxoplamosis, an important zoonotic parasitic disease, and thus causes serious public safety issues (Montoya and Liesenfeld, 2004; Chen et al., 2012a; Tian et al., 2012; Zhang et al., 2013). Although $T$. gondii infections in immunocompetent individuals are subclinical or usually asymptomatic, the infection can cause severe clinical problem or death in immunosuppressed and immunodeficient individuals, such as Toxoplasma encephalitis (TE) or neurological lesions (Montoya and Liesenfeld, 2004; Weiss and Dubey, 2009; Dubey, 2010). In animals, the parasite is responsible for considerable economic losses in the livestock industry resulting from abortion or congenital toxoplasmosis in all types of livestock, especially in sheep and goats (Fayer et al., 2004; Dubey et al., 2005).

T. gondii heat shock protein 60 (TgHSP60) is a member of the HSP60 subfamily (Toursel et al., 2000), which plays an important role in intracellular survival and in the differentiation of $T$. gondii and also mediates its virulence (Shonhai et al., 2011). In addition, TgHSP60 not only serves as a signal for the activation of antigen presenting cells by inducing the release of cytokines and stimulation of the initial immune response, but also acts as a molecular chaperone in the mitochondria (Ma et al., 2009). Regardless of the key biological roles of TgHSP60 mentioned above, no previous studies have elucidated the sequence diversity of the HSP60 gene among T. gondii isolates from different origins. Therefore, the objectives of the present study were to examine sequence variation in the entire coding region of the hsp60 gene among strains from different geographical regions and hosts, and to assess whether the locus may be used as a new marker for population genetic studies of $T$. gondii isolates.

\section{MATERIAL AND METHODS}

\section{T. gondii isolates}

Five T. gondii isolates from different geographical locations and hosts were used for the analysis in this study (Table 1) (Zhou et al., 2009, 2010; Su et al., 2010; Huang et al., 2012). All isolates were harvested according to the method described by Yan et al. (2011), and total RNA was extracted using the Total RNA Kit I (Omega; USA) according to manufacturer recommendations. The total RNA obtained was stored at $-80^{\circ} \mathrm{C}$ until use. 
Table 1. Details of Toxoplasma gondii isolates used.

\begin{tabular}{llll}
\hline Isolate ID & Host & Geographical location & Genotype* \\
\hline TgPLh & Human & Shanghai, China & Type I, ToxoDB 10 \\
QHO & Sheep & Huzhu, Qinghai, China & Type II, ToxoDB 1 \\
Prugniaud (PRU) & Human & France & Type II, ToxoDB 1 \\
PYS & Pig & Panyu, Guangdong, China & Type \#3, ToxoDB 9 \\
TgC7 & Cat & Guangzhou, Guangdong, China & Type \#3, ToxoDB 9 \\
\hline *Based on genotyping results of Zhou et al. (2009, 2010), Su et al. (2010), and Huang et al. (2012).
\end{tabular}

\section{Polymerase chain reaction (PCR) amplification}

A pair of specific primers (forward primer HSP60F:5'-ATGCTTGCCCGCGCTTCAGC3'; reverse primer HSP60R: 5'-CTAGTACATGCCTCCCATGCCGC-3') were designed to amplify the TgHSP60 gene based on the HSP60 gene sequence of the T. gondii ME49 strain available in the National Center for Biotechnology Information (NCBI; GenBank accession number XM002367081.1). Reverse transcription (RT)-PCR amplification was performed using the PrimeScript ${ }^{\mathbb{B}}$ One Step RT-PCR Kit Ver.2 (TAKARA) according to manufacturer recommendations. RT-PCR were performed in a volume of $25 \mu \mathrm{L}$ consisting of $1 \mu \mathrm{L}$ PrimeScript 1 step enzyme mix, $12.5 \mu \mathrm{L} 2 \mathrm{X} 1$ Step buffer, $2.5 \mu \mathrm{M}$ of each primer, 100-200 ng total RNA, and $1 \mu \mathrm{L}$ RNase-free $\mathrm{dH}_{2} \mathrm{O}$. The thermal cycling conditions were as follows: 1) reverse transcription at $50^{\circ} \mathrm{C}$ for $30 \mathrm{~min}$; 2) RTase inactivation at $94^{\circ} \mathrm{C}$ for $2 \mathrm{~min}$; 3) denaturation at $94^{\circ} \mathrm{C}$ for $1 \mathrm{~min}$, annealing at $60.4^{\circ} \mathrm{C}$ for $45 \mathrm{~s}$, and extension at $72^{\circ} \mathrm{C}$ for 2 min for 30 cycles each; and 4) additional extension for $10 \mathrm{~min}$ at $72^{\circ} \mathrm{C}$. The amplified products were electrophoresed on $1 \%(\mathrm{w} / \mathrm{v})$ agarose gels, stained with GoldenView ${ }^{\mathrm{TM}}$, and photographed using a gel documentation system (UVP GelDoc-It ${ }^{\mathrm{TM}}$ Imaging System; Cambridge, UK). The DL2000 marker (TAKARA) was utilized to estimate the sizes of the hsp60 RT-PCR products.

\section{Sequencing of the $h \operatorname{sp} 60$ amplicons, sequence analysis, and phylogenetic reconstruction}

The hsp60 PCR products were purified using spin columns (Wizard ${ }^{\mathrm{TM}}$ PCR-Preps DNA Purification System; Promega; USA), ligated with the pMD18-T easy vector (TAKARA) according to manufacturer recommendations, and then transformed into Escherichia coli DH5 $\alpha$ competent cells (Promega). The positive colonies were screened by PCR amplification and enzymatic digestion, and were sequenced by the Shanghai Songon Biological Engineering Biotechnology Company.

The sequences obtained were aligned using ClustalX 1.83 (Thompson et al., 1997), and the intraspecific sequence variation was calculated by pair-wise sequence comparison using the formula $D=1-(M / L)$, where $M$ is the same number of sequence bases and $L$ is the total number of sequence bases. Phylogenetic reconstructions based on the entire coding region of the $h s p 60$ gene among the different $T$. gondii strains were performed using maximum parsimony (MP) and Bayesian inference (BI), using Neospora caninum (Gene ID: NCLIV_065210) as an outgroup. MP analysis was performed using PAUP* 4.0b10 (Swofford, 2002), with indels being treated as missing character states. A total of 100 random addition searches using tree bisection-reconnection (TBR) branch swapping were performed for 
each MP analysis. Bootstrap probability (BP) was calculated from 1000 bootstrap replicates with 10 random additions per replicate in PAUP. BI analyses were conducted with four independent Markov chains run for 1,000,000 metropolis-coupled Markov chain Monte Carlo (MCMC) generations, sampling a tree every 1000 generations in MrBayes 3.1.1 (Ronquist and Huelsenbeck, 2003).

\section{RESULTS AND DISCUSSION}

The length of the coding sequence of the hsp 60 gene was 1728 bp for all of the examined T. gondii strains, and the $\mathrm{A}+\mathrm{T}$ contents varied from 41.96 to $42.13 \%$. Sequence variations in the entire coding region of $h s p 60$ among the examined $T$. gondii strains were $0-1.44 \%$, which is similar to that observed in the ROP7, ROP13, and MIC13 genes (Ren et al., 2012; Wang et al., 2012; Zhou et al., 2012). The alignment of all the 8 sequences revealed 20 variable positions, showing $1.16 \%$ overall sequence variation, which suggested a relatively considerable sequence diversity, but a lower genetic variation than that of the coding regions of the GRA5, GRA6, and AK69 genes (Fazaeli et al., 2000; Boughattas et al., 2011; Chen et al., 2012b). Of these variable positions, there were 15 transitions ( $\mathrm{A} \leftrightarrow \mathrm{G}$, and $\mathrm{C} \leftrightarrow \mathrm{T})$ and 5 transversions ( $\mathrm{A} \leftrightarrow \mathrm{C}, \mathrm{A} \leftrightarrow \mathrm{T}, \mathrm{G} \leftrightarrow \mathrm{C}$, and $\mathrm{G} \leftrightarrow \mathrm{T})$ in the coding region of the T. gondii hsp60 gene ( $\mathrm{R}$ $=$ transition $/$ transversion $=3$ ).

Phylogenetic analysis using BI and MP based on the $h s p 60$ coding sequences showed that $T$. gondii strains representing genotype I and genotype II, the two major clonal lineage types, were grouped into their respective clusters separately, and the ToxoDB \#9 strains could be readily distinguished from the classical genotypes (Figure 1). This result is consistent with that of some previous studies using other genetic markers such as GRA5, GRA6, and AK69 for genotyping (Fazaeli et al., 2000; Boughattas et al., 2011; Chen et al., 2012b).

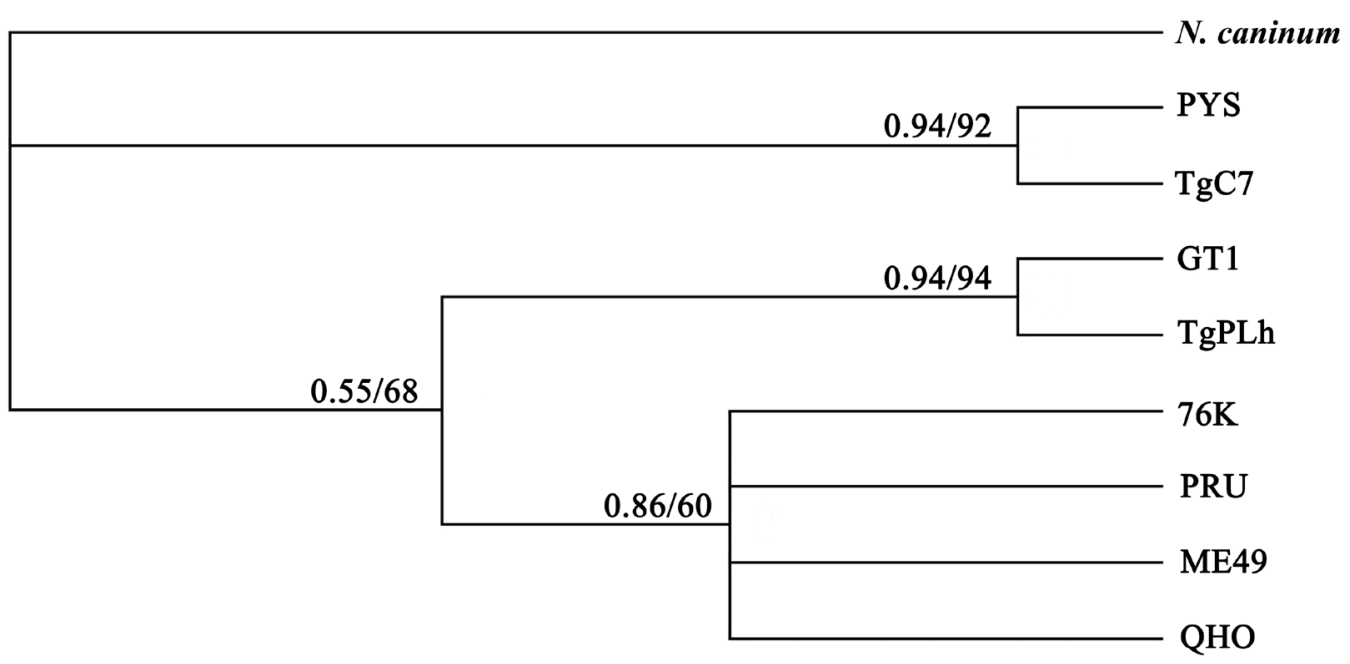

Figure 1. Phylogram of 8 Toxoplasma gondii strains determined by analysis of the entire sequences of the hsp60 gene sequences. The high genetic divergence of the hsp60 gene revealed two major (denoted by I and II) and atypical ToxoDB 9. The tree was built by Bayesian inference (BI) and maximum parsimony (MP) analysis. The numbers along branches indicate bootstrap values resulting from different analysis in the order: BI/MP. 
In conclusion, the present study determined the entire coding sequences of the $T$. gondii hsp60 gene and revealed relatively considerable sequence variability in this locus among $T$. gondii isolates from different hosts and geographical regions. Phylogenetic analysis suggested that the $T$. gondii $h s p 60$ coding sequence may represent a novel genetic marker for studying the population diversity and molecular epidemiology of $T$. gondii.

\section{Conflicts of interest}

The authors declare no conflict of interest.

\section{ACKNOWLEDGMENTS}

Research partially supported by the National Natural Science Foundation of China (Grant \#31228022, \#31230073, and \#31172316), the International Science and Technology Cooperation Project of Gansu Province (Grant \#1204WCGA023), and The Science Fund for Creative Research Groups of Gansu Province (Grant \#1210RJIA006).

\section{REFERENCES}

Boughattas S, Ben-Abdallah R, Siala E, Ben-Abda I, et al. (2011). Tunisian Toxoplasma gondii strains genotyping by the use of AK69 marker. Parasit. Vectors. 4: 167.

Chen J, Xu MJ, Zhou DH, Song HQ, et al. (2012a). Canine and feline parasitic zoonoses in China. Parasit. Vectors. 5: 152.

Chen J, Li ZY, Zhou DH, Liu GH, et al. (2012b). Genetic diversity among Toxoplasma gondii strains from different hosts and geographical regions revealed by sequence analysis of GRA5 gene. Parasit. Vectors. 5: 279.

Dubey JP (2010). Toxoplasmosis of Animals and Humans. 2nd edn. CRC Press Inc., Boca Raton.

Dubey JP, Hill DE, Jones JL, Hightower AW, et al. (2005). Prevalence of viable Toxoplasma gondii in beef, chicken, and pork from retail meat stores in the United States: risk assessment to consumers. J. Parasitol. 91: 1082-1093.

Fayer R, Dubey JP and Lindsay DS (2004). Zoonotic protozoa: from land to sea. Trends Parasitol. 20: 531-536.

Fazaeli A, Carter PE, Darde ML and Pennington TH (2000). Molecular typing of Toxoplasma gondii strains by GRA6 gene sequence analysis. Int. J. Parasitol. 30: 637-642.

Huang SY, Cong W, Zhou P, Zhou DH, et al. (2012). First report of genotyping of Toxoplasma gondii isolates from wild birds in China. J. Parasitol. 98: 681-682.

Ma GY, Zhang JZ, Yin GR, Zhang JH, et al. (2009). Toxoplasma gondii: proteomic analysis of antigenicity of soluble tachyzoite antigen. Exp. Parasitol. 122: 41-46.

Montoya JG and Liesenfeld O (2004). Toxoplasmosis. Lancet 363: 1965-1976.

Ren D, Zhou DH, Xu MJ and Zhou Y (2012). Sequence variation in Toxoplasma gondii MIC13 gene among isolates from different hosts and geographical locations. Afr. J. Microbiol. Res. 6: 1333-1337.

Ronquist F and Huelsenbeck JP (2003). MrBayes 3: Bayesian phylogenetic inference under mixed models. Bioinformatics 19: $1572-1574$.

Shonhai A, Maier AG, Przyborski JM and Blatch GL (2011). Intracellular protozoan parasites of humans: the role of molecular chaperones in development and pathogenesis. Protein Pept. Lett. 18: 143-157.

Su C, Shwab EK, Zhou P, Zhu XQ, et al. (2010). Moving towards an integrated approach to molecular detection and identification of Toxoplasma gondii. Parasitology 137: 1-11.

Swofford DL (2002). Paup*: Phylogenetic Analysis Using Parsimony, Version 4.0b10. Sinauer Associates, Sunderland.

Thompson JD, Gibson TJ, Plewniak F, Jeanmougin F, et al. (1997). The CLUSTAL_X windows interface: flexible strategies for multiple sequence alignment aided by quality analysis tools. Nucleic Acids Res. 25: 4876-4882.

Tian YM, Dai FY, Huang SY, Deng ZH, et al. (2012). First report of Toxoplasma gondii seroprevalence in peafowls in Yunnan Province, Southwestern China. Parasit. Vectors. 5: 205.

Toursel C, Dzierszinski F, Bernigaud A, Mortuaire M, et al. (2000). Molecular cloning, organellar targeting and developmental expression of mitochondrial chaperone HSP60 in Toxoplasma gondii. Mol. Biochem. Parasitol. 111: 319-332. 
Wang PY, Lu P, Xu MJ and Li J (2012). Genetic diversity among Toxoplasma gondii isolates from different hosts and geographical locations revealed by analysis of ROP13 gene sequences. Afr. J. Biotechnol. 11: 6662-6665.

Weiss LM and Dubey JP (2009). Toxoplasmosis: A history of clinical observations. Int. J. Parasitol. 39: 895-901.

Yan HK, Song HQ, Zhou Y and Ren D (2011). Sequence variation in perforin-Like protein 1 gene among six Toxoplasma gondii strains. J. Anim. Vet. Adv. 10: 2244-2247.

Zhang NZ, Chen J, Wang M, Petersen E, et al. (2013). Vaccines against Toxoplasma gondii: new developments and perspectives. Expert. Rev. Vaccines. 12: 1287-1299.

Zhou Y, Lu P, Xu MJ and Ren D (2012). Sequence variation in TgROP7 gene among Toxoplasma gondii isolates from different hosts and geographical regions. Afr. J. Biotechnol. 11: 6658-6661.

Zhou P, Zhang H, Lin RQ, Zhang DL, et al. (2009). Genetic characterization of Toxoplasma gondii isolates from China. Parasitol. Int. 58: 193-195.

Zhou P, Nie H, Zhang LX, Wang HY, et al. (2010). Genetic characterization of Toxoplasma gondii isolates from pigs in China. J. Parasitol. 96: 1027-1029. 